ARTICLE

Received 2 Apr 2013 | Accepted 25 Nov 2013 | Published 9 Jan $2014 \quad$ DOl: 10.1038/ncomms4012 OPEN

\title{
Non-classicality of the molecular vibrations assisting exciton energy transfer at room temperature
}

\author{
Edward J. O'Reilly ${ }^{1} \&$ Alexandra Olaya-Castro ${ }^{1}$
}

Advancing the debate on quantum effects in light-initiated reactions in biology requires clear identification of non-classical features that these processes can exhibit and utilize. Here we show that in prototype dimers present in a variety of photosynthetic antennae, efficient vibration-assisted energy transfer in the sub-picosecond timescale and at room temperature can manifest and benefit from non-classical fluctuations of collective pigment motions. Non-classicality of initially thermalized vibrations is induced via coherent exciton-vibration interactions and is unambiguously indicated by negativities in the phase-space quasiprobability distribution of the effective collective mode coupled to the electronic dynamics. These quantum effects can be prompted upon incoherent input of excitation. Our results therefore suggest that investigation of the non-classical properties of vibrational motions assisting excitation and charge transport, photoreception and chemical sensing processes could be a touchstone for revealing a role for non-trivial quantum phenomena in biology.

\footnotetext{
${ }^{1}$ Department of Physics and Astronomy, University College London, Gower Street, London WC1E 6BT, UK. Correspondence and requests for materials should be addressed to A.O.-C. (email: a.olaya@ucl.ac.uk).
} 
T he experimental demonstration of oscillatory electronic dynamics in light-harvesting complexes ${ }^{1-5}$ has triggered wide-spread interest in uncovering quantum phenomena that may have an impact on the function of the molecular components of living organisms. In general, however, oscillatory patterns in dynamics are not sufficient argument to rule out classical descriptions of the same behaviour. Indeed, recent work has discussed how classical coherence models can predict electronic coherence beating ${ }^{6,7}$. Therefore, an important challenge for the growing field of quantum effects in biomolecules is to clearly identify which quantum features with no classical counterpart may manifest in these systems and how they may influence the process of interest.

The question of non-classicality of the dynamics of electronic excitations in light-harvesting systems has been addressed by investigating Leggett-Garg inequalities ${ }^{8}$. This work concludes that, under Markovian evolution, temporal correlations of the observables of individual pigments should violate classical bounds, and in consequence certain classical theories are unsuitable to describe electronic dynamics. Other works have investigated the quantumness of the electronic degrees of freedom ${ }^{9-13}$. Despite these efforts, it is still far from understood which non-classical phenomena are directly correlated with efficient energy distribution in a prototype light-harvesting system.

What is clear is that exciton energy transport depends not only on the topology of electronic couplings among pigments but is critically determined by exciton-phonon interactions: molecular motions $^{14}$ and environmental fluctuations ${ }^{14-16}$ drive efficient transport processes in light-harvesting antennae. In fact, it is well known that exciton-phonon interactions in these complexes have a rich structure as a function of energy and generally include coupling to both continuous and discrete modes associated to low-energy solvated protein fluctuations and underdamped intramolecular vibrations, respectively ${ }^{14}$. Moreover, evidence is mounting that the interaction between excitons and underdamped vibrations whose energies commensurate exciton splittings may be at the heart of the coherence beating probed in two-dimensional (2D) photon echo spectroscopy ${ }^{17-23}$. Although some insights into the importance of such resonances can be gained from Förster theory ${ }^{24}$, the wider implications for optimal spatio-temporal distribution of energy ${ }^{19,25,26}$, for modulation of exciton coherences ${ }^{19-22}$ and for collective pigment motion dynamics ${ }^{23}$ have just recently started to be clarified.

The current state of the debate then suggests that a conceptual advance in understanding non-trivial quantum phenomena assisting excitation transport could emerge precisely from investigating non-classical features of the molecular motions and phonon environments that play such a key role. Techniques able to manipulate vibrational states ${ }^{27,28}$ and probe their quantum properties ${ }^{29,30}$ may indeed provide the experimental platform to address this issue. Here we investigate this question in a prototype dimer ubiquitous in light-harvesting antennae of cyanobacteria $^{31}$, cryptophyte algae ${ }^{32,33}$ and higher plants ${ }^{34-36}$ and show that commensurate energies of exciton splitting and underdamped high-energy vibrations allows exciton-vibration dynamics to induce and harness non-classical fluctuations of collective pigment motions for efficient energy transfer. Negative values of the Mandel Q-parameter ${ }^{37}$ indicating sub-Poissonian phonon occupation fluctuations and, correspondingly, negative regions in a regularized quasi-probability $P$ distribution in phase space $^{38,39}$ unambiguously preclude any classical description of such fluctuations or its correlations with transport. Our results show a potential functional relevance of non-classicality of molecular fluctuations for exciton transport and therefore provide a framework to investigate similar non-trivial quantum phenomena in the large variety of biomolecular transport ${ }^{40,41}$, photoreception $^{42}$ and chemical sensing processes ${ }^{43-45}$ that are known (or hypothesized) to be assisted by unequilibrated vibrational motion.

\section{Results}

Characterizing non-classicality. The field of quantum optics has developed a solid framework to quantify the quantum properties of bosonic fields ${ }^{46}$. It therefore provides excellent conceptual and quantitative tools to investigate non-classicality of the harmonic vibrational degrees of freedom of interest in this work. From the perspective of quantum optics, quantum behaviour with no classical counterpart-that is, non-classicality-arises if the state of the system of interest cannot be expressed as a statistical mixture of coherent states defining a valid probability measure ${ }^{47}$ This then leads to non-positive values of a phase-space quasi-probability distribution such as the Glauber-Sudarshan $P(\alpha)$-function ${ }^{47}$.

$$
P(\alpha)=\frac{1}{\pi^{2}} \int d^{2} \xi e^{\alpha^{*}-\alpha^{*} \xi} \chi(\xi),
$$

where $\chi(\xi)$ is the characteristic function of the bosonic quantum state. However, highly singular behaviour of $P(\alpha)$ can make its characterization challenging both theoretically and experimentally. To overcome this, verification of the non-classicality of a quantum state can be performed by constructing a regularized distribution $P_{w}(\alpha)$ as the Fourier transform of a filtered quantum characteristic function $\chi_{w}(\xi)^{38,39}$ as explained in the Methods section. Negativities in this regularized distribution are necessary and sufficient condition of quantum behaviour with no classical analogue $e^{38}$ and offer a significant advantage over other distributions, such as the Wigner distribution, which can be positive for quantum states that are truly non-classical.

As an alternative to phase-space distributions, signatures of non-classicality can be observed in the fluctuations of the bosonic field. For a single mode, negative values of the Mandel's Q-parameter ${ }^{37}$ can be a signature of non-classical behaviour. It characterizes the departure of the occupation number distribution $P(n)$ from Poissonian statistics through the inequality

$$
Q=\frac{\left\langle\hat{n}^{2}\right\rangle-\langle\hat{n}\rangle^{2}}{\langle\hat{n}\rangle}-1<0
$$

where $\langle\hat{n}\rangle$ and $\left\langle\hat{n}^{2}\right\rangle$ denote the first and second moments of the bosonic number operator $\hat{n}$, respectively. Vanishing $Q$ indicates Poissonian number statistics where the mean of $\hat{n}$ equals its variance as it is characteristic of classical wave-like behaviourthat is, a coherent state of light. For a chaotic thermal state, one finds that $Q=\langle\hat{n}\rangle>0$ indicating that particles are 'bunched'. A Fock state is characterized by $Q<0$ indicating that particle occupation is restricted to a particular level. Inequalities involving occupation probabilities of nearest number states can similarly witness non-classical occupation fluctuations ${ }^{48}$.

In what follows we use the above framework to investigate the non-classical behaviour of the vibrational motions that drive excitation dynamics in prototype dimers present in a variety of antennae proteins of photosynthetic systems.

Prototype dimers and collective motion. We consider a prototype dimer where each chromophore has an excited electronic state of energy $\varepsilon_{i}$ strongly coupled to a quantized vibrational mode of frequency $\omega_{\text {vib }}$ much larger than the thermal energy scale $K_{\mathrm{B}} T$ and described by the bare Hamiltonians $H_{\mathrm{el}}=\sum_{i=1,2}$ $\varepsilon_{i} \sigma_{i}^{+} \sigma_{i}^{-}$and $H_{\mathrm{vib}}=\omega_{\mathrm{vib}}\left(b_{i}^{\dagger} b_{1}+b_{2}^{\dagger} b_{2}\right)$, respectively. Inter-chromophore coupling is generated by dipole-dipole interactions of the form $H_{\mathrm{d}-\mathrm{d}}=V\left(\sigma_{1}^{+} \sigma_{2}^{-}+\sigma_{2}^{+} \sigma_{1}^{-}\right)$. The electronic excited states interact with their local vibrational environments with 
strength $g$, linearly displacing the corresponding mode coordinate, $H_{\mathrm{el}-\mathrm{vib}}=g \sum_{i=1,2} \sigma_{i}^{+} \sigma_{i}^{-}\left(b_{i}^{\dagger}+b_{i}\right)$. In the above $b_{i}^{\dagger}\left(b_{i}\right)$ creates (annihilates) a phonon of the vibrational mode of chromophore $i$, while $\sigma_{i}^{ \pm}$creates or destroys an electronic excitation at site $i$. The eigenstates $|X\rangle$ and $|Y\rangle$ of $H_{\mathrm{el}}+H_{\mathrm{d}-\mathrm{d}}$ denote exciton states with energy splitting given by $\Delta E=\left((\Delta \varepsilon)^{2}+4 V^{2}\right)^{1 / 2}$ and $\Delta \varepsilon=\varepsilon_{1}-\varepsilon_{2}$. Transformation into collective mode coordinates shows that centre of mass mode $b_{\mathrm{cm}}^{(\dagger)}=\left(b_{1}^{(\dagger)}+b_{2}^{(\dagger)}\right) / \sqrt{2}$ decouples from the electronic degrees of freedom and that only the mode corresponding to the relative displacement mode with bosonic operators

$$
b_{\mathrm{rd}}^{(\dagger)}=\left(b_{1}^{(\dagger)}-b_{2}^{(\dagger)}\right) / \sqrt{2}
$$

couples to the excitonic system. It is the non-classical properties of this collective mode that we investigate. In collective coordinates, the effective exciton-vibration Hamiltonian then reads

$$
H_{\mathrm{ex}-\mathrm{vib}}=\frac{\Delta \varepsilon}{2} \sigma_{z}+V \sigma_{x}-\frac{g}{\sqrt{2}} \sigma_{z}\left(b_{\mathrm{rd}}^{\dagger}+b_{\mathrm{rd}}\right)+\omega_{\mathrm{vib}} b_{\mathrm{rd}}^{\dagger} b_{\mathrm{rd}},
$$

with $\sigma_{z}=\sigma_{2}^{+} \sigma_{2}^{-}-\sigma_{1}^{+} \sigma_{1}^{-}$and $\sigma_{x}=\left(\sigma_{1}^{+} \sigma_{2}^{-}+\sigma_{2}^{+} \sigma_{1}^{-}\right)$. Tiwary et al. ${ }^{23}$ have recently pointed out that $2 \mathrm{D}$ spectroscopy can probe the involvement of these anticorrelated, relative displacement motions in electronic dynamics. From now on and for simplicity, we denominate this relative displacement mode as the collective mode.

We are interested in dimers that satisfy $\Delta E \sim \omega_{\text {vib }}>g>V$ where the effects of underdamped high-energy vibrational motions are expected to be most important ${ }^{19,31,36}$. Several natural light-harvesting antennae include pairs of chromophores that clearly fall in this regime. Two important examples of such dimers are illustrated in Fig. 1a,b and correspond to the central PEB (phycoeritrobilin) ${ }_{50 c}-\mathrm{PEB}_{50 d}$ dimer in the cryptophyte antennae PE545 (ref. 33) and a $\mathrm{Chl}_{b 601}$ - $\mathrm{Chl}_{a 602}$ pair in the light-harvesting complex II (LHCII) complex of higher plants ${ }^{36}$; both corresponding to systems that have exhibited coherence beating in $2 \mathrm{D}$ spectroscopy $\mathrm{y}^{3,4,49}$. Importantly, in each case, the dimer considered contributes to an important energy transfer pathway towards exit sites ${ }^{3,33}$, suggesting that the the phenomena we discuss will have an effect in the performance of the whole complex. Moreover, synthetic versions of such prototype dimers could be available ${ }^{50}$. Most remarkably, LHCII is likely the most abundant light-harvesting complex on Earth ${ }^{35}$, while cryptophyte antennae such as PE545 are ecologically important as they support photosynthesis under extreme low-light conditions ${ }^{51,52}$. From this perspective, the dimers of interest are exceptionally relevant biomolecular prototypes. Spectroscopy studies indicate that these dimers are subject to a structured exciton-phonon interaction as considered in our model. For the $\mathrm{PEB}_{50}$ dimer, the intramolecular mode of interest has frequency around $1,111 \mathrm{~cm}^{-1}$ (ref. 33), which compares with the frequency of the breathing mode of the tetrapyrrole ${ }^{53}$ (Carles Curutchet, personal communication). In the case of the $\mathrm{Chl}_{b-a}$ pair, it has been shown that a mode around $750 \mathrm{~cm}^{-1}$ is coupled to the electronic dynamics ${ }^{36}$ and this energy is close to the frequency of in-plane deformations of the pyrrole ${ }^{54}$. Furthermore, vibrational dephasing in chromophores $^{55}$ and in other systems such as photoreceptors ${ }^{56}$ is known to be of the order of picoseconds. Some aspects of the influence of non-equilibrium vibrational motion in these specific dimers have been considered before ${ }^{19,57}$; however, none of these studies have addressed the question of interest: can vibrationassisted transport exploit quantum phenomena that have no classical analogue?

Non-classicality via coherent exciton-vibration dynamics. We first consider the quantum coherent dynamics associated to $H_{\mathrm{ex}-\mathrm{vib}}$ to illustrate how non-classical behaviour of the collective motion emerges out of an initial thermal phonon distribution and an excitonic state with no initial superpositions: $\rho\left(t_{0}\right)=|X\rangle\langle X| \otimes \rho_{\mathrm{vib}}^{\mathrm{th}}$, which in the basis of exciton-vibration states of the form $|X, n\rangle$ (see Fig. 2a), becomes $\rho\left(t_{0}\right)=\sum_{n} P_{\mathrm{th}}(n)|X, n\rangle\langle X, n|$. Here $n$ denotes the phonon occupation number of the relative displacement mode coupled to exciton dynamics (see Eq. 4), while $P_{\mathrm{th}}(n)$ denotes the thermal occupation of such level. The observables of interest are the

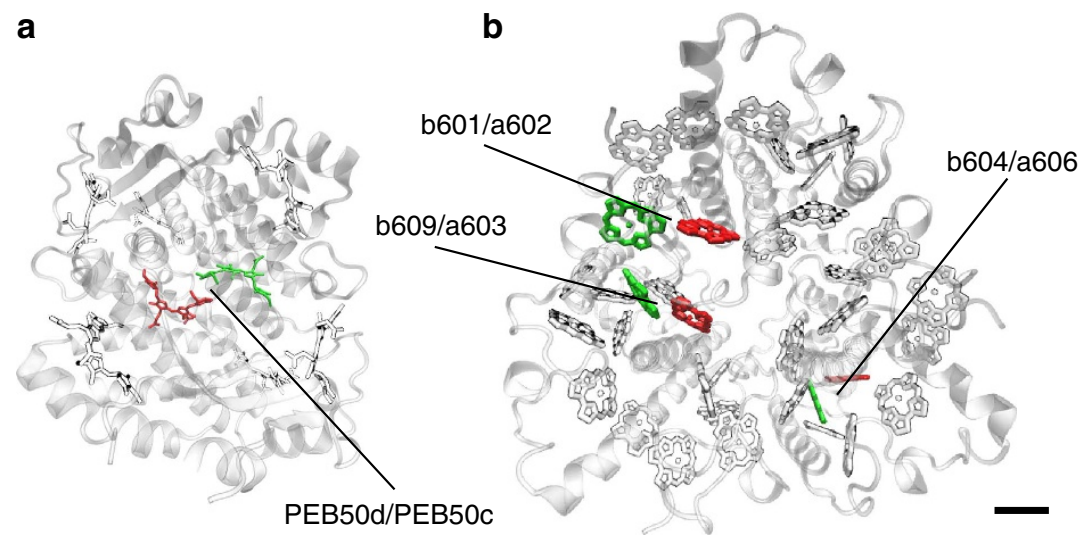

Figure 1 | Prototype dimers. (a,b) Cryptophyte antennae phycoerythrin 545 (PE545) and LHCII present in higher plants have pairs of pigments whose electronic and vibrational parameters fall in the regime of our vibration-assisted transport model. (a) Representation of the pigments and protein environment of a PE545 complex of Rhodomonas CS24 (Protein Data Bank ID code 1XG0, ref. 32). The central PEB dimer pigments PEB 50 c and PEB 50 are highlighted in red and green, respectively. For this $\mathrm{PEB}_{50}$ dimer, there is an uncertainty in the value of the energy gap 32,33 . We take parameters from refs 32,33 such that $\Delta \varepsilon=1,042 \mathrm{~cm}^{-1}$ and $V=92 \mathrm{~cm}^{-1}$, so $\Delta E=1,058.2 \mathrm{~cm}^{-1}$ being quasi-resonant with an intramolecular mode of frequency

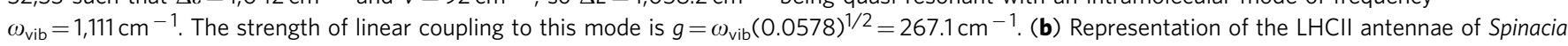
oleracea (Protein Data Bank ID code 1RWT, ref. 34). Several pairs of close $\mathrm{Chl}_{b}$-Chl $($ red-green) chlorophylls satisfy the conditions of our model. In particular, we consider the $\mathrm{Chl}_{b 601}-\mathrm{Chl}_{a 602}$ pair for which $\Delta \varepsilon=661 \mathrm{~cm}^{-1}$ and $V=-47.1 \mathrm{~cm}^{-1}$, resulting in $\Delta E=667.7 \mathrm{~cm}^{-1}$ (ref. 66 ). An intramolecular vibrational mode of frequency $\omega_{\text {vib }}=742.0 \mathrm{~cm}^{-1}$ is close to this energy gap and each chromophore couples to this mode with strength $g=\omega_{\text {vib }}(0.03942)^{1 / 2}=147.3 \mathrm{~cm}^{-1}$ as obtained from (ref. 40). Scale bar: $1 \mathrm{~nm}$. 
population of the lowest excitonic state $\rho_{Y Y}(t)=\sum_{n}$ $\langle Y, n|\rho(t)| Y, n\rangle$, the absolute value of the coherence $\rho_{X 0-Y 0}(t)$ $=\langle X, 0|\rho(t)| Y, 0\rangle$, which denotes the inter-exciton coherence in the ground state of the collective vibrational mode, and the non-
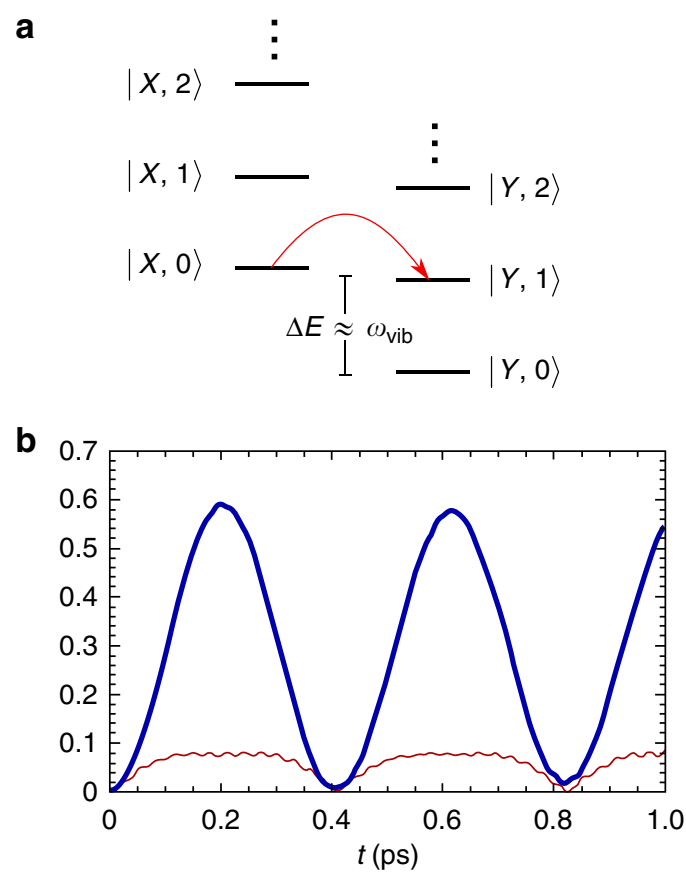

Figure 2 | Exciton-collective mode states and free exciton dynamics.

(a) The energy levels of the exciton-collective mode states used to describe energy transfer $|X, n\rangle$. The red arrow denotes population transfer from $|X, 0\rangle$ to $|Y, 1\rangle$. (b) Quantum coherent dynamics of the PEB 50 dimer in PE545 illustrating population of the lowest exciton $\rho_{Y Y}(t)$ (thick blue curve) and the inter-exciton coherence in the ground-state of the collective mode $\left|\rho_{X O-Y_{O}}(t)\right|$ (thin red curve). classicality given by negative values $Q(t)$ and corresponding negativities in the regularized quasi-probability distribution $P_{w}(\alpha)$. Hamiltonian evolution generates coherent transitions from states $|X, n\rangle$ to $|Y, n+1\rangle$ (see Fig. 2a) with a rate $f$ that depends on the exciton delocalization $(|V| / \Delta \varepsilon)$, the coupling to the mode $g$ and the phonon occupation $n$-that is, $f \simeq g(2|V| / \Delta \varepsilon) \sqrt{(n+1) / 2}$. Since $\omega_{\text {vib }} \gg K_{\mathrm{B}} T$ the ground state of the collective mode is largely populated, such that the Hamiltonian evolution of the initial state is dominated by the evolution of the state $|X, 0\rangle$. This implies that the energetically close exciton-vibration state $|Y, 1\rangle$ becomes coherently populated at a rate $f \simeq g(2|V| / \Delta \varepsilon)$, leading to the oscillatory pattern observed in the probability of occupation $\rho_{Y Y}(t)$ as illustrated in Fig. 2b. The low-frequency oscillations of the dynamics of $\rho_{Y Y}(t)$ cannot be assigned to the exciton or the vibrational degrees of freedom alone as expected from quantum coherent evolution of the exciton-plus-effective mode system. For instance, if the mode occupation is restricted to at most $n=1$, the period of the amplitude of $\rho_{Y Y}(t)$ is given approximately by the inverse of

$$
\frac{1}{2}\left(\sqrt{(\Delta E-\alpha)^{2}+\frac{2 g^{2} 4 V^{2}}{\alpha \Delta E}}-(\Delta E+\alpha)\right),
$$

with $\alpha^{2}=2 g^{2}+\omega_{\text {vib }}^{2}$ and $\left(2 g^{2} 4 V^{2} / \alpha^{2} \Delta E^{2}\right) \ll 1$. Coherent exciton population transfer is accompanied by beating of the inter-exciton coherence $\left|\rho_{X 0-Y 0}(t)\right|$, with the main amplitude modulated by the same low-frequency oscillations of $\rho_{Y Y}(t)$ and a superimposed fast oscillatory component of frequency close to $\omega_{\text {vib }}$ (see Fig. 2b). This fast-driving component arises from local oscillatory displacements: when $V \simeq 0$ the time evolution of each local mode is determined by the displacement operator with amplitude $\alpha(t)=2 g\left(1-\exp \left(-i \omega_{\text {vib }} t\right)\right) / \omega_{\text {vib }}$ (ref. 58). As the state $|Y, 1\rangle$ is coherently populated, the collective quantized mode is driven out of equilibrium towards a non-classical state in which selective occupation of the first vibrational level takes place, thereby modulating occupation of higher levels. This manifests itself in sub-Poissonian phonon statistics as indicated by negative values of $Q(t)$ shown in Fig. 3a. Similar phenomena have been described
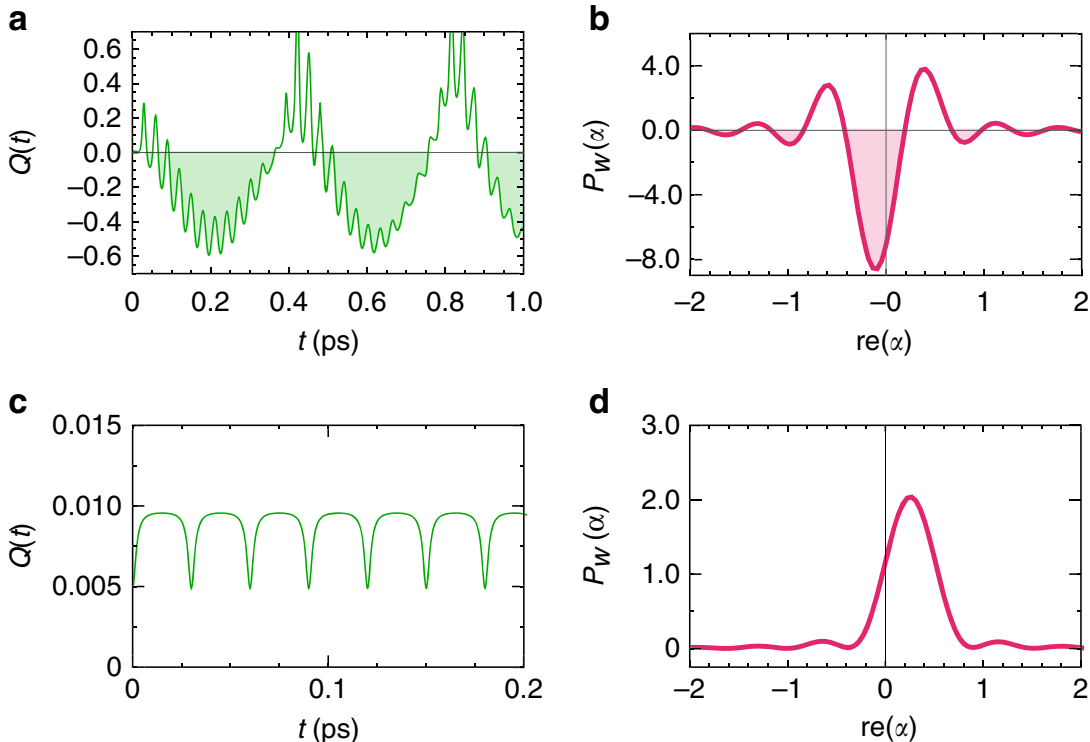

Figure 3 | Non-classicality of collective and local vibrational modes. (a) Mandel Q-parameter of the relative displacement mode of the considered PEB 50 dimer from PE545 when a biological electronic coupling is considered. Shaded regions denote times of non-classicality. (b) The associated regularized quasi-probability distribution $P_{\mathrm{w}}(\alpha)$ at $t=0.2 \mathrm{ps}$. Shaded regions denote areas of negative probability. (c) Mandel Q-parameter of the intramolecular highenergy vibration when dipole coupling is zero and (d) the associated regularized quasi-probability distributions $P_{\mathrm{w}}(\alpha)$ at $t=0.2$ ps. 
in the context of electron transport in a nanoelectromechanical system $^{59,60}$. Moreover, Fig. $3 \mathrm{~b}$ shows that at times when $Q(t)$ is negative-that is, $t=0.2 \mathrm{ps}$-the regularized quasi-probability distribution $P_{w}(\alpha)$ at this time exhibits negativities, thereby ruling out any classical description of the same phenomena. Interestingly, the non-classical properties of the collective vibrational motion resemble non-classicality of bosonic thermal states (completely incoherent states) that are excited by a single quanta ${ }^{39,61}$. Importantly, such non-classical behaviour of the vibrational motion arises only when the electronic interaction between pigments is finite. For comparison, Fig. $3 \mathrm{c}$ shows that if $V=0$, an electronic excitation drives the local underdamped vibration towards a thermal displaced state with super-Poissonian statistics $(Q(t)>0)$, which has an associated positive probability distribution in phase space as illustrated in Fig. 3d. In short, nonclassicality of the collective mode quasi-resonant with the excitonic transition arises through the transient formation of exciton-vibration states.

Energy and coherence dynamics under thermal relaxation. We now investigate the dynamics of the exciton-vibration dimer when each local electronic excitation interacts additionally with a low-energy thermal bath described by a continuous distribution of harmonic modes. The strength of this interaction is described by a Drude spectral density with associated reorganization energy $\lambda$ and cutoff frequency $\Omega_{\mathrm{c}}<K_{\mathrm{B}} T$ as described in the Methods section. We consider the exciton and vibration parameters to the $\mathrm{PEB}_{50}$ dimer and investigate the trends as functions of the reorganization energy. As expected, the interplay between vibrationactivated dynamics and thermal fluctuations leads to two distinct regimes of energy transport as a function of $\lambda$. For our consideration of weak electronic coupling, the coherent transport regime is determined approximately by $\left(\lambda \Omega_{\mathrm{c}}\right)^{1 / 2} \leq 2 g|V| / \Delta \epsilon$. Population of the low-lying exciton state is dominated by coherent transitions between exciton-collective mode states and the rapid, non-exponential growth of $\rho_{Y Y}(t)$ in this regime can be traced back to coherent evolution from $|X, 0\rangle$ to $|Y, 1\rangle$. At longer timescales thermal fluctuations induce incoherent transitions from $|X, 0\rangle$ to $|Y, 0\rangle$ with a rate proportional to $\left(\lambda \Omega_{c}\right)^{1 / 2}$, thereby stabilizing population of $\rho_{Y Y}(t)$ to a particular value as can be seen in Fig. 4d. This behaviour is illustrative of what is expected in the dimer $\mathrm{Chl}_{b-\mathrm{a}}$ for which $\lambda=37 \mathrm{~cm}^{-1}$ as obtained from ${ }^{40}$. To confirm this we have computed the exciton-vibration dynamics with parameters of the $\mathrm{Chl}_{b-a}$ dimer, the results of which are shown in Supplementary Fig. S1. In contrast, for $\left(\lambda \Omega_{c}\right)^{1 / 2}>2 g|V| / \Delta \epsilon$ population transfer to $\rho_{Y Y}(t)$ is incoherent. For the $\mathrm{PEB}_{50}$ dimer $\lambda \sim 110 \mathrm{~cm}^{-1}$ that place this dimer in this incoherent regime where $\rho_{Y Y}(t)$ has a slow but continuous exponential rise reflecting the fact that thermal fluctuations inducing transitions from $|X, n\rangle$ to $|Y, n\rangle$ now have a large contribution to exciton transport. However, even in this regime, transfer to $\rho_{Y Y}(t)$ is always more efficient with the quasi-resonant mode than in the situations where only thermal-bath-induced transitions are considered (see dashed lines in Fig. $4 \mathrm{a}, \mathrm{d}, \mathrm{g}$ ). The underlying reason is that before vibrational relaxation takes place (around $t=1 \mathrm{ps}$ ), the system is transiently evolving towards a thermal configuration of exciton-collective mode states. Hence, in both coherent and incoherent population transfer regimes, transfer to the lowest exciton state involves a transient, selective population of the first vibrational level of the collective mode. The transition from coherent to incoherent exciton population dynamics is then marked by the onset of energy dissipation of the exciton-vibration system as shown in Fig. 4c,f,i, where $E(t)=\operatorname{Tr}\left\{H_{\mathrm{ex}-\mathrm{vib}} \rho(t)\right\}$ has been depicted for different values of $\lambda$. While exciton population growth is non-exponential, energy dissipation into the thermal bath is transiently prevented as indicated by periods of positive slope of $E(t)$ as happens in Fig. 4c,f. Quantification of the energy that is transiently 'extracted' from the low-energy thermal bath can provide an interesting physical interpretation of the advantages of non-exponential exciton transfer in the framework of non-equilibrium thermodynamics ${ }^{62}$.

For completeness, we present in Figure $4 \mathrm{~b}, \mathrm{e}, \mathrm{h}$ how the beating patterns of the coherence $\rho_{X 0-Y 0}(t)$ reveal the structured nature of the exciton-phonon interaction and witnesses whether there is coherent exciton-vibration evolution as it has been pointed out by recent studies ${ }^{21,22}$. The frequency components of such oscillatory exciton coherences vary depending on the coupling to the thermal bath. In the coherent regime, as $\rho_{X 0-Y_{0}}(t)$ follows exciton populations, the main amplitude is modulated by the same relevant energy difference between exciton-vibration states (see Fig. 4b,e). This behaviour is relevant for the parameter regime of the $\mathrm{Chl}_{b-a}$ dimer (see Supplementary Fig. S1). In contrast, for the $\mathrm{PEB}_{50}$ dimer, the short-time oscillations of $\rho_{X 0-Y 0}(t)$ (between $t=0$ and $t=0.1 \mathrm{ps}$ ) arise from purely electronic correlations because of bath-induced renormalization of the electronic Hamiltonian ${ }^{63}$. This exciton coherence retains the superimposed driving at a frequency $\omega_{\text {vib }}$ and is accompanied by non-classicality as it will be described shortly, indicating that vibrational motion is still out of thermal equilibrium. The dynamical features presented in Fig. 4g,h agree with previous findings based on a perturbative approach ${ }^{19}$ and with the timescales of the exciton coherence beating reported for cryptophyte algae $e^{4,18}$.

Non-classicality under thermal relaxation. Interaction with the thermal environment would eventually lead to the emergence of classicality in longer timescales. However, in the picosecond timescale of interest, the collective mode exhibits periods of nonclassicality across a wide range of thermal bath couplings $\lambda$ as indicated by sub-Possonian fluctuations with $Q(t)<0$ in Fig. 5a-c and the corresponding negativities in the distributions $P_{w}(\alpha)$ shown in Fig. 5d-f. This survival of non-classicality is concomitant with a slow decay of the exciton-vibration coherence $\rho_{X 0, Y 1}(t)$ (not shown). Non-classical behaviour of collective fluctuations are then expected for the parameters of both the $\mathrm{PEB}_{50}$ dimer for which $\lambda=110 \mathrm{~cm}^{-1}$ and the $\mathrm{Chl}_{b-a}$ dimer for which $\lambda=37 \mathrm{~cm}^{-1}$. The non-classical fluctuations predicted by $Q(t)$ also agree with those witnessed by a parameter quantifying correlations between nearest-neighbours' occupations ${ }^{48}$, which we present in Supplementary Fig. S2. As expected, the maximum non-classicality indicated by the most negative value of $Q(t)$ decreases for larger reorganization energies. Nonetheless, the time average of these non-classicalities is not a monotonic function of $\lambda$. For moderate values of $\lambda$, the collective mode spends longer periods in states with non-classical fluctuations-that is, periods for which $Q(t)<0$ as seen in Fig. 5b, thereby stabilizing nonclassicality at a particular level. This sub-picosecond stabilization of non-classicality is expected in the regime of the $\mathrm{Chl}_{b-a}$ dimers as illustrated in Supplementary Fig. S1.

Functional role of non-classicality. Non-classical fluctuations of collective motions correlate to exciton population transfer. In order to demonstrate this, we investigate quantitative relations between non-classicality and exciton energy transport by considering relevant integrated averages in the timescale of the Hamiltonian evolution of the exciton-vibration system denoted by $\tau$. For the parameters of the $\mathrm{PEB}_{50}$, this timescale is about half a picosecond and is comparable to the timescale in which excitation energy would be distributed away to other chromophores 
a

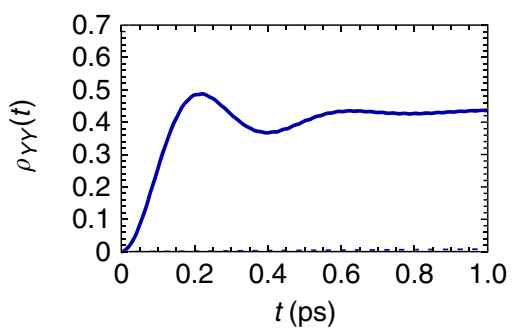

b

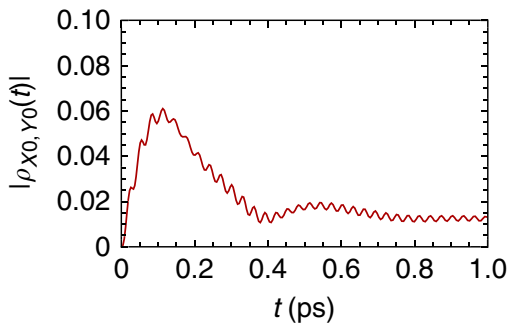

c

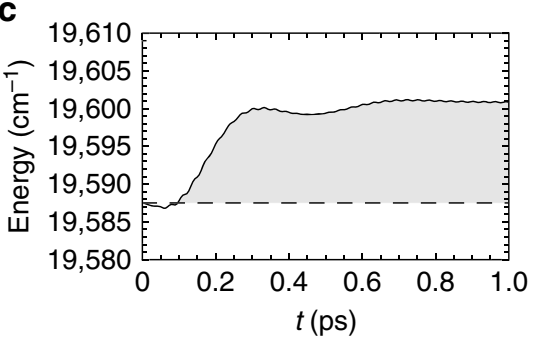

d
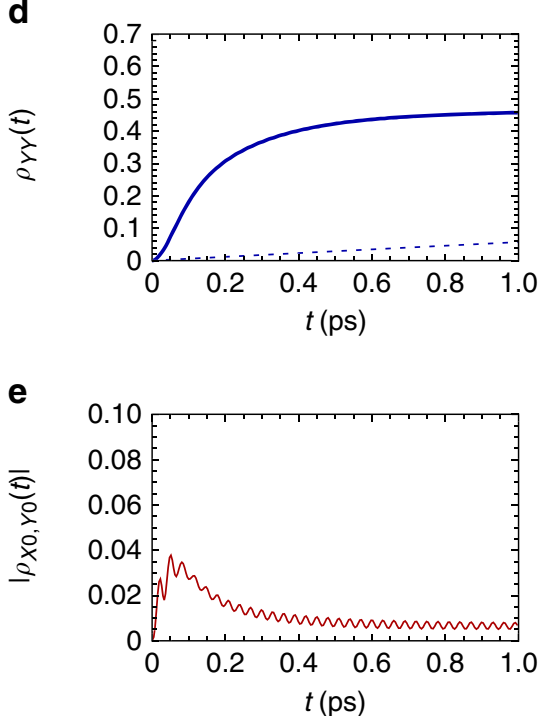

f

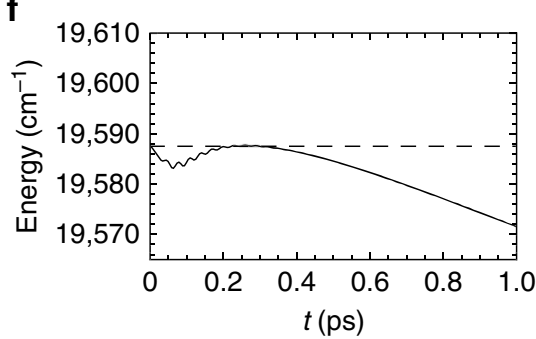

g

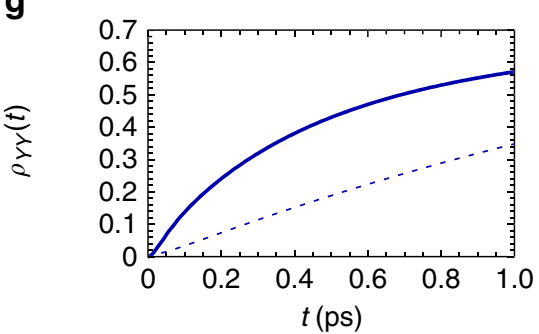

h

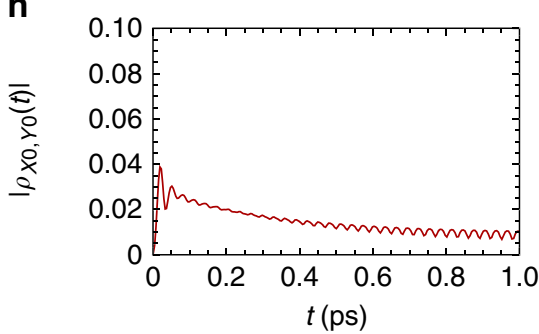

i

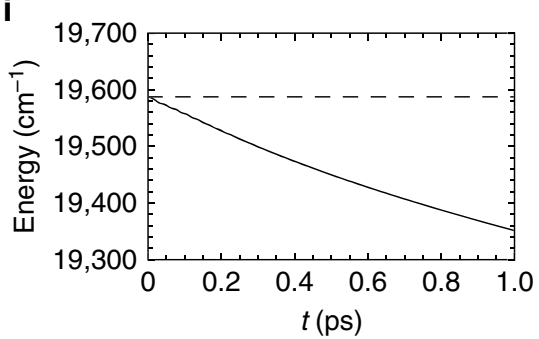

Figure 4 I Energy and coherence evolution under thermal relaxation. The dynamics of $\rho_{Y Y}(t)$ with (thick blue curve) and without (dashed curve) coupling to vibration (top row), $\left|\rho_{X \mathrm{O}, Y_{0}}(t)\right|$ (middle row) and energy of the exciton vibration system $E(t)=\operatorname{Tr}\left\{H_{\mathrm{ex}}-\mathrm{vib} \rho(t)\right\}$ (bottom row) for the exciton-vibration parameters of the $\mathrm{PEB}_{50}$ dimer and three interaction strengths to the low-energy thermal bath: $(\mathbf{a}-\mathbf{c}): \lambda=6 \mathrm{~cm}-1,(\mathbf{d}-\mathbf{f}) \lambda=35 \mathrm{~cm}-1$ and $(\mathbf{g}-\mathbf{i}): \lambda=110 \mathrm{~cm}^{-1}$. Initial energy $E(0)$, displayed as dashed line and times where $E(t)>E(0)$ are shaded.
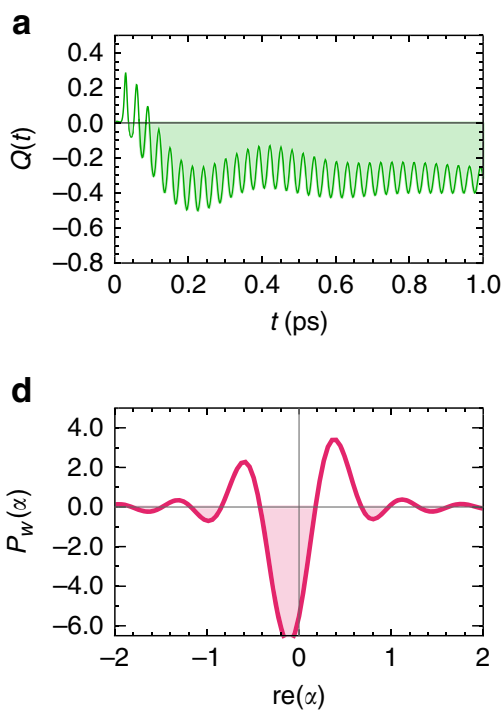

b
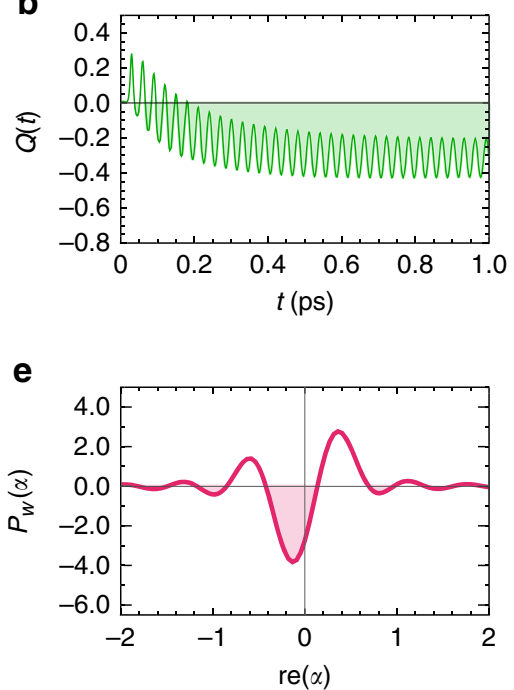

c

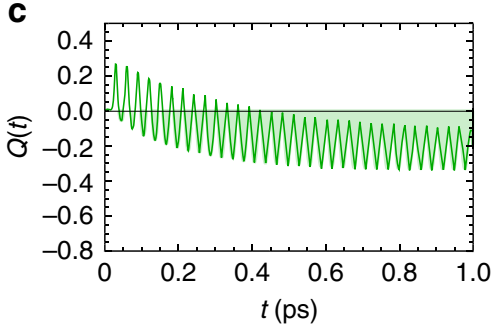

f

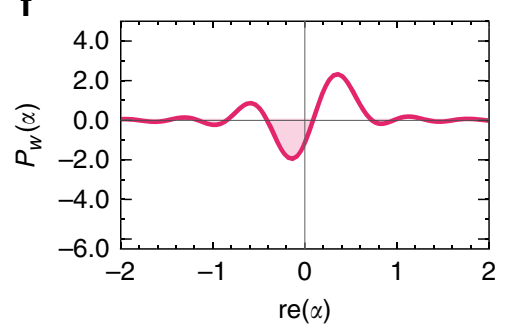

Figure 5 | Non-classicality of collective motions under thermal relaxation. (a-c) Dynamics of the Mandel Q-parameter for $\lambda=6,35$, and $110 \mathrm{~cm}^{-1}$, respectively. Shaded regions denote times of non-classicality. (d-f) Regularized distribution $P_{w}(\alpha)$ at $t=0.2$ ps for each corresponding value of $\lambda$. Shaded regions denote areas of negative probability.

or to a trapping state. The time-integrated averages over $\tau$ are defined as: $\langle F[\rho(t)]\rangle_{\tau}=(1 / \tau) \int_{0}^{\tau} \mathrm{d} t F[\rho(t)]$, where $F[\rho(t)]$ corresponds to the exciton population $\rho_{Y Y}(t)$ and the non- classicality of the underdamped collective mode through periods of sub-Poissonian statistics $Q(t) \Theta[-Q(t)]$ as functions of the coupling to the bath $\lambda$. As shown in Fig. 6 , the average exciton 


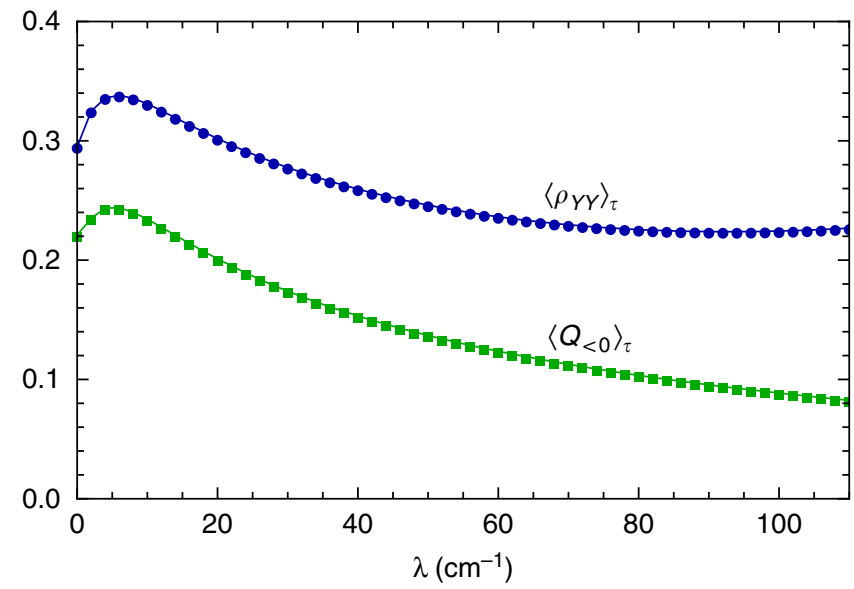

Figure 6 | Correlations between non-classicality and exciton populations. Time-integrated averages of exciton population $\rho_{Y Y}(t)$ and non-classicality as quantified by $Q(t) \Theta[-Q(t)]$ (blue and green, respectively) as functions of coupling to the thermal background by fixing environment cutoff frequency $\Omega_{\mathrm{c}}=100 \mathrm{~cm}^{-1}$ and varying reorganization energy $\lambda$ with exciton-vibration parameters corresponding to the $\mathrm{PEB}_{50}$ dimer.

population and non-classicality follow a similar non-monotonic trend as a function of the coupling to the thermal bath, indicating a direct quantitative relation between efficient energy transfer in the timescale $\tau$ and the degree of non-classicality. The appearance of a maximal point in the average non-classicality as a function of the system-bath coupling indicates that the average quantum response of the collective anticorrelated motion to the impulsive electronic excitation is optimal for a small amount of thermal noise.

It is worth emphasizing that the above functional role of nonclassicality holds for vibration-assisted transport where the highenergy intramolecular modes considered are quasi-resonant with the excitonic energy splitting. When vibrational motions are significantly detuned with the bare exciton transition $\Delta E$, transport is dominated by the thermal background and no selective population of the state $|Y, 1\rangle$ takes place; hence, periods of sub-Poissonian fluctuations vanish. To illustrate the difference with the off-resonance case, Fig. 7 shows the same timeintegrated averages as in Fig. 6, with the electronic parameters of the $\mathrm{PEB}_{50}$ dimer but now considering an intramolecular vibration of frequency $\omega_{\text {vib }}=1,520 \mathrm{~cm}^{-1}$ significantly detuned from $\Delta E$. In this case, thermally activated transport (see dashed line in Fig. 7) and vibration-assisted transport (solid line in Fig. 7) are practically indistinguishable and the average $Q(t)$ is positive with a value near zero as expected for a thermal distribution of a high-energy harmonic mode.

The degree of purity of the initial exciton state is also important in enabling and harnessing non-classical fluctuations of the collective mode. One therefore should expect that statistical mixtures of excitons with finite purity can still trigger such nonclassical response. To illustrate this, we now consider mixed initial states of the form $\rho\left(t_{0}\right)=\rho_{\mathrm{ex}} \otimes \rho_{\mathrm{vib}}^{\mathrm{th}}$ where $\rho_{\mathrm{ex}}=r|X\rangle\langle X|$ $-(1-r)|Y\rangle\langle Y|$ with $1 / 2 \leq r \leq 1$. The associated linear entropy quantifying the mixedness of the initial exciton states is given by $S_{\mathrm{L}}=2 r(r-1)$. The time-averaged non-classicality (Fig. 8a) and average population transfer (Fig. 8b) follow similar decreasing, yet non-zero, monotonic trends for mixed states. These results suggest that non-classical vibrational motion can be prompted and exploited even under incoherent conditions creating statistical mixture of excitons ${ }^{64}$. The trends presented in Figs 6,8 constitute theoretical evidence of direct quantitative

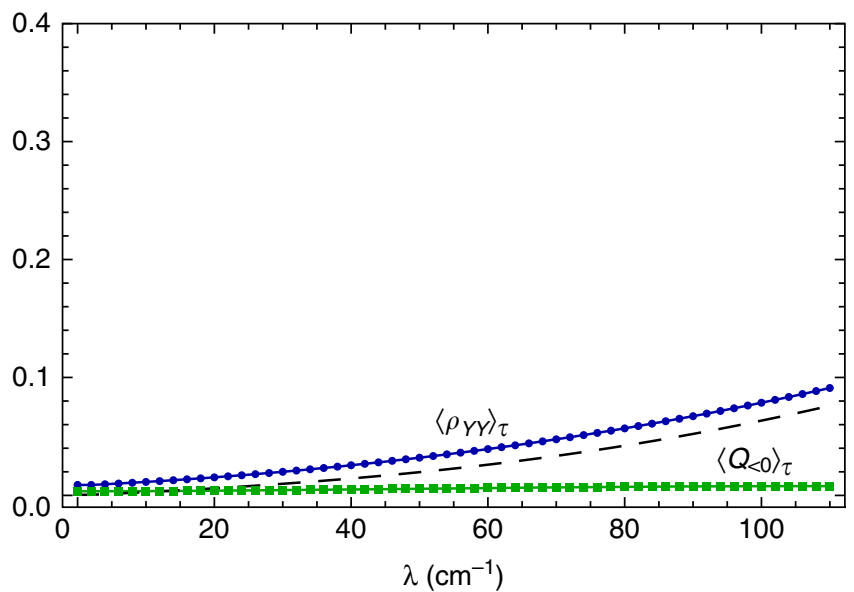

Figure 7 | Effects of off-resonance vibrations. Time-integrated averages as in Fig. 6 with electronic parameters of the $\mathrm{PEB}_{50}$ dimer but now considering an intramolecular vibration which is $\omega_{\text {vib }}=1,520 \mathrm{~cm}^{-1}$-that is, significantly detuned from the energy gap $\Delta E=1,058.2 \mathrm{~cm}^{-1}$ and similar coupling strength $g=\omega_{\text {vib }}(0.0265)^{1 / 2} \approx 247 \mathrm{~cm}^{-1}$. Parameters obtained from ref. 33. The dashed curve shows time average of $\rho_{Y Y}(t)$ when no intramolecular vibration is considered and transport results only because of the thermal background.
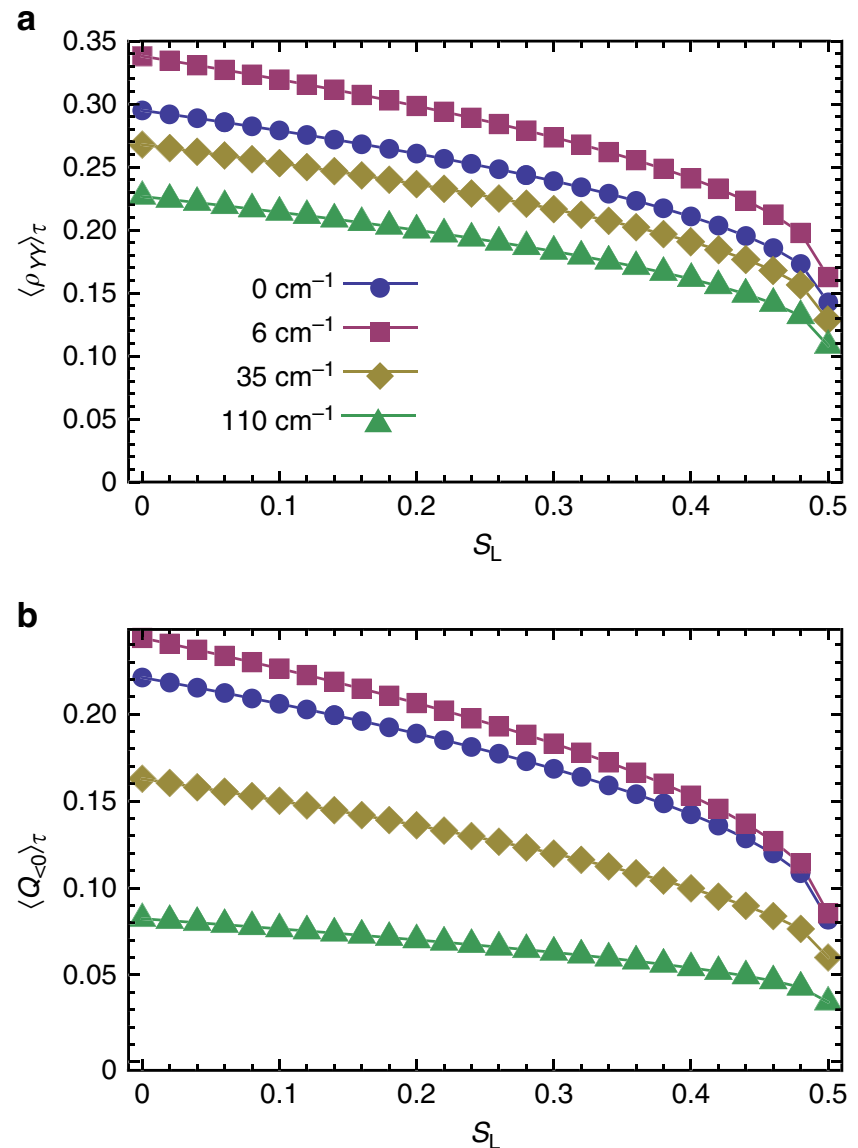

Figure 8 | Non-classicality under incoherent exciton input. The timeintegrated averages of exciton population $\rho_{Y Y}(t)-\rho_{Y Y}(0)$ and nonclassicality as quantified by $Q(t) \Theta[-Q(t)]$ as functions of the linear entropy $S_{L}=2 r(r-1)$ for $0<r<1 / 2$. Curves are for different system-bath couplings. 
correlations between non-classicality and exciton population transport in a relevant sub-picosecond timescale and therefore illustrate a functional role for quantum phenomena with no classical counterpart in a prototype light-harvesting system. Our results remain valid when the picosecond-dephasing rate of the vibrational motion is included in the dynamics as can be seen in Supplementary Fig. S3.

\section{Discussion}

Light-harvesting complexes are fundamental components of the photosynthetic machinery on Earth. While there has been an extraordinary advance in the techniques to probe these systems at ultrafast timescales, it is conceptually unclear what truly quantum features with no classical counterpart these systems may exhibit and exploit for efficient energy transport ${ }^{65}$. Besides depending on long-range electronic interactions, excitation energy distribution is fundamentally affected by the molecular motions and harmonic (or in cases anharmonic) environments that modulate the electronic dynamics. We therefore put forward the idea that, precisely, investigation of non-classical phenomena associated to such molecular motions can pave the way towards understanding which non-trivial quantum phenomena can have an impact on efficient energy distribution and trapping. Within this line of thought, we investigate non-classical behaviour in a prototype dimer that conveys fundamental physical principles of vibrationassisted transport in a variety of light-harvesting antennae. We demonstrate that quasi-resonances between excitonic transitions and underdamped high-energy intramolecular vibrations can trigger and harness non-trivial quantum behaviour of collective pigment motions that are initially in a thermal, fully incoherent, state. In this scenario, correlations between non-classical fluctuations of collective pigment motions and efficient population of the target exciton state are found. Negative values both of the Mandel Q-parameter and of the quasi-probability distribution $P_{w}(\alpha)$ of the collective motion assure that no classical distribution can describe the same behaviour. These non-trivial quantum phenomena are predicted for a variety of initial excitation conditions including statistical mixtures of excitons indicating that such non-classicality can be activated even under incoherent input of photoexcitations. Transient coherent ultrafast phonon spectroscopy ${ }^{29}$, which is sensitive to low-phonon populations of high-energy vibrations ${ }^{30}$, may provide an interesting experimental approach to probe the phenomena we describe.

The prototype exciton-vibration dimers here investigated are representative of interband-like transfer pathways present in the majority of light-harvesting complexes. For instance, in the LHCII complex, the considered dimer contributes to the fastest component of the $\mathrm{Chl}_{b} \rightarrow \mathrm{Chl}_{a}$ interband transfer pathway that directs excitation energy towards exit sites ${ }^{66}$. The demonstration that non-classicality is concomitant with efficient vibrationassisted transfer in these dimers therefore suggests that nonclassical phenomena will have a contribution to the efficiency of the whole complex. A rigorous quantitative estimation of such contribution requires both a careful extension of our formalism to quantify these features in a multi-modal system (as likely other non-equilibrated vibrational motion will be involved) and a careful weighting of the vibration-assisted processes in the overall spatio-temporal distribution of energy.

The framework we propose can also be applied to gain insights into the non-classical response of vibrational motion in a variety of transport ${ }^{40,41}$ and sensing processes in biomolecules ${ }^{42,43}$. Of particular interest are charge transfer in reaction centres ${ }^{40}$ and isomerization of photoreceptors ${ }^{42}$ where specific intramolecular vibrational motions are known to be driven out of thermal equilibrium during the light-initiated electronic dynamics. It will also be interesting to use this framework to understand possible non-trivial quantum behaviour of molecular motions in chemical sensors ${ }^{43}$ that are conjectured to operate through weak electronic interactions to sense molecular vibrations of the order of a thousand wavenumbers 44,45

We have also illustrated how in our prototype dimer with biologically relevant parameters, exciton-vibration dynamics can lead to non-exponential excitonic energy distribution whereby dissipation into a low-energy thermal bath can be transiently prevented. From this view, coherent vibrational motions that do not relax quickly and whose fluctuations cannot be described classically may be seen as an internal quantum mechanism controlling energy distribution and storage. Further insights into the advantage of these non-trivial quantum behaviour may therefore be gained in a thermodynamic framework ${ }^{62,67}$.

In conclusion, we have provided theoretical evidence that vibration-assisted exciton transport in prototype dimers, representative of interband-like transitions in a variety of photosythetic light-harvesting antennae, can exploit non-trivial quantum phenomena that cannot be reproduced by any classical counterpart, namely, non-classical fluctuations of collective pigment motions. Given that a variety of transport ${ }^{40,41}$ and sensing phenomena $^{42}$ in biomolecules are known to involve nonequilibrium vibrational motion, our findings have broad implications for the field of quantum effects in biology as they suggest that investigating the non-classical nature of molecular fluctuations harnessed in these processes could be the key to reveal a role for truly non-trivial quantum features.

\section{Methods}

Open quantum system dynamics. To accurately account for the effects of the low-energy thermal bath, we have adopted a hierarchical expansion of the excitonvibration dynamics ${ }^{68-70}$. We split the high-energy mode from the bath of harmonic oscillators and explicitly treat the quantum interactions between electronic excitations and these modes of frequency $\omega_{\text {vib }}$ by including it within the definition of the system, $H_{\mathrm{ex}-\mathrm{vib}}=H_{\mathrm{ex}} \otimes \mathbb{1}_{\mathrm{vib}}+\mathbb{1}_{\mathrm{ex}} \otimes H_{\mathrm{vib}}+H_{\mathrm{ex}-\mathrm{vib}}$. The electronic operators then couple to the remaining vibrational modes $H_{\mathrm{I}}=\sum_{i \mathbf{k}} g_{i}\left(\sigma_{i}^{+} \sigma_{i}^{-} \otimes 1_{\mathrm{vib}}\right)\left(b_{\mathbf{k}}^{\dot{\mathbf{k}}}+b_{\mathbf{k}}\right)$. This approach allows the effects of the lowenergy thermal bath on the exciton-vibration dynamics to be accurately accounted for. Truncating the quantized mode at Fock level $M=6$ adequately describes both reduced dynamics of the collective quantized mode and the electronic dynamics of the prototype dimer at room temperature. A spectral density of the form $J(\omega)=2 \lambda \Omega_{\mathrm{c}} \omega /\left(\Omega_{\mathrm{c}}^{2}+\omega^{2}\right)$ is assumed, where $\lambda$ and $\Omega_{\mathrm{c}}$ are the reorganization energy and cutoff frequency, respectively. Supplementary Note 1 furnishes further details of the hierarchical expansion of exciton-vibration dynamics. Converged dynamics are obtained by terminating the hierarchical expansion at level $N=11$ and including just the $K=0$ Matsubara term. No additional Matsubara terms are necessary as $\Omega_{\mathrm{c}}<K_{\mathrm{B}} T$. For completeness, we present dynamics including the $K=1$ term in Supplementary Fig. S4.

Regularized quasi-probability distributions. The quasi-probability distribution we calculate is a regularized version of the $P$-representation

$P_{w}(\alpha)=\left(1 / \pi^{2}\right) \int d^{2} \xi e^{\alpha \xi^{*}-\alpha^{*} \xi} \chi(\xi) \Omega_{w}(\xi)$, where the quantum characteristic function $\chi(\xi)=\operatorname{Tr}\left\{e^{\xi b_{\mathrm{rd}}^{+}-\xi^{*} b_{\mathrm{rd}}} \rho_{\text {vib }}\right\}$ of the reduced state the vibration $\rho_{\text {vib }}$ is reconstructed in the truncated Fock basis $\left\langle m\left|\rho_{\text {vib }}\right| n\right\rangle . \Omega_{w}(\xi)$ is a non-classicality filter ${ }^{38}$ taken as the triangular function with $w=5$, which fulfils the necessary condition of a filter such that $P_{w}(\xi)$ detects non-classicality. More details are given in Supplementary Note 2 .

\section{References}

1. Engel, G. S. et al. Evidence for wavelike energy transfer through quantum coherence in photosynthetic systems. Nature 446, 782-786 (2007).

2. Panitchayangkoon, G. et al. Long-lived quantum coherence in photosynthetic complexes at physiological temperature. Proc. Natl Acad. Sci. USA 107, 12766-12770 (2010)

3. Calhoun, T. R. et al. Quantum coherence enabled determination of the energy landscape in light-harvesting complex II. J. Phys. Chem. B 113, 16291-16295 (2009).

4. Collini, E. et al. Coherently wired light-harvesting in photosynthetic marine algae at ambient temperature. Nature 463, 644-647 (2010). 
5. Harel, E. \& Engel, G. S. Quantum coherence spectroscopy reveals complex dynamics in bacterial light-harvesting complex 2 (LH2). Proc. Natl Acad. Sci. USA 109, 706-711 (2012).

6. Miller, W. H. Perspective: quantum or classical coherence? J. Chem. Phys. 136, 210901 (2012)

7. Briggs, J. S. \& Eisfeld, A. Equivalence of quantum and classical coherence in electronic energy transfer. Phys. Rev. E 83, 051911 (2011).

8. Wilde, M. M., McCracken, J. M. \& Mizel, A. Could light harvesting complexes exhibit non-classical effects at room temperature? Proc. R. Soc. A 466, 1347-1363 (2010)

9. Olaya-Castro, A., Lee, C. F., Olsen, F. F. \& Johnson, N. F. Efficiency of energy transfer in a light-harvesting system under quantum coherence. Phys. Rev. B 78, 085115 (2008).

10. Sarovar, M., Ishizaki, A., Fleming, G. R. \& Whaley, K. B. Quantum entanglement in photosynthetic light-harvesting complexes. Nat. Phys. 6, 462-467 (2010)

11. Caruso, F., Chin, A. W., Datta, A., Huelga, S. F. \& Plenio, M. B. Entanglement and entangling power of the dynamics in light-harvesting complexes. Phys. Rev. A 81, 062346 (2010).

12. Fassioli, F. \& Olaya-Castro, A. Distribution of entanglement in light-harvesting complexes and their quantum efficiency. New J. Phys. 12, 085006 (2010).

13. Whaley, K. B., Sarovar, M. \& Ishizaki, A. Quantum entanglement phenomena in photosynthetic light harvesting complexes. Procedia Chem. 3, 152-164 (2011).

14. Renger, T., May, V. \& Kühn, O. Ultrafast excitation energy transfer dynamics in photosynthetic pigment-protein complexes. Phys. Rep. 343, 137-254 (2001).

15. Plenio, M. B. \& Huelga, S. F. Dephasing-assisted transport: quantum networks and biomolecules. New J. Phys. 10, 113019 (2008).

16. Rebentrost, P., Mohseni, M., Kassal, I., Lloyd, S. \& Aspuru-Guzik, A. Environment-assisted quantum transport. New J. Phys. 11, 033003 (2009).

17. Richards, G. H., Wilk, K. E., Curmi, P. M. G., Quiney, H. M. \& Davis, J. A. Coherent vibronic coupling in light-harvesting complexes from photosynthetic marine algae. J. Phys. Chem. Lett. 3, 272-277 (2012).

18. Turner, D. B. et al. Quantitative investigations of quantum coherence for a light-harvesting protein at conditions simulating photosynthesis. Phys. Chem. Chem. Phys. 14, 4857-4874 (2012).

19. Kolli, A., O’Reilly, E. J., Scholes, G. D. \& Olaya-Castro, A. The fundamental role of quantized vibrations in coherent light harvesting by cryptophyte algae. J. Chem. Phys. 137, 174109 (2012).

20. Chin, A. W., Huelga, S. F. \& Plenio, M. B. Coherence and decoherence in biological systems: principles of noise-assisted transport and the origin of longlived coherences. Philos. Trans. R. Soc. A 370, 3638-3657 (2012).

21. Chin, A. W. et al. The role of non-equilibrium vibrational structures in electronic coherence and recoherence in pigment-protein complexes. Nat. Phys. 9, 113-118 (2013)

22. Christensson, N., Kauffmann, H. F., Pullerits, T. \& Mancăl, T. Origin of long-lived coherences in light-harvesting complexes. J. Phys. Chem. B 116, 7449-7454 (2012)

23. Tiwari, V., Peters, W. K. \& Jonas, D. M. Electronic resonance with anticorrelated pigment vibrations drives photosynthetic energy transfer outside the adiabatic framework. Proc. Natl Acad. Sci. USA 110, 1203-1208 (2013).

24. Förster, T. 10th spiers memorial lecture. Transfer mechanisms of electronic excitation. Discuss. Faraday Soc. 27, 7-17 (1959).

25. Perdomo, A., Vogt, L., Najmaie, A. \& Aspuru-Guzik, A. Engineering directed excitonic energy transfer. Appl. Phys. Lett. 96, 093114 (2010).

26. Rey, M. d., Chin, A. W., Huelga, S. F. \& Plenio, M. B. Exploiting structured environments for efficient energy transfer: the phonon antenna mechanism. J. Phys. Chem. Lett. 4, 903-907 (2013).

27. Biggs, J. D. \& Cina, J. A. Using wave-packet interferometry to monitor the external vibrational control of electronic excitation transfer. J. Chem. Phys. 131, 224101 (2009)

28. Biggs, J. D. \& Cina, J. A. Studies of impulsive vibrational influence on ultrafast electronic excitation transfer. J. Phys. Chem. A 116, 1683-1693 (2012).

29. Waldermann, F. C. et al. Measuring phonon dephasing with ultrafast pulses using raman spectral interference. Phys. Rev. B 78, 155201 (2008).

30. Lee, K. C. et al. Entangling macroscopic diamonds at room temperature. Science 334, 1253-1256 (2011).

31. Womick, J. M. \& Moran, A. M. Vibronic enhancement of exciton sizes and energy transport in photosynthetic complexes. J. Phys. Chem. B 115, 1347-1356 (2011).

32. Doust, A. B. et al. Developing a structure-function model for the cryptophyte phycoerythrin 545 using ultrahigh resolution crystallography and ultrafast laser spectroscopy. J. Mol. Biol. 344, 135-153 (2004).

33. Novoderezhkin, V. I., Doust, A. B., Curutchet, C., Scholes, G. D. \& van Grondelle, R. Excitation dynamics in phycoerythrin 545: modeling of steady-state spectra and transient absorption with modified redfield theory. Biophys. J. 99, 344-352 (2010)
34. Liu, Z. et al. Crystal structure of spinach major light-harvesting complex at 2.72 Å resolution. Nature 428, 287-292 (2004).

35. Barros, T. \& Kühlbrandt, W. Crystallisation, structure and function of plant light-harvesting complex II. Biochim. Biophys. Acta Bioenerg. 1787, 753-772 (2009).

36. Novoderezhkin, V. I., Palacios, M. A., van Amerongen, H. \& van Grondelle, R Energy-transfer dynamics in the LHCII complex of higher plants:modified redfield approach. J. Phys. Chem. B 108, 10363-10375 (2004).

37. Mandel, L. Sub-poissonian photon statistics in resonance fluorescence. Opt. Lett. 4, 205-207 (1979).

38. Kiesel, T. \& Vogel, W. Nonclassicality filters and quasiprobabilities. Phys. Rev. A 82, 032107 (2010).

39. Kiesel, T., Vogel, W., Bellini, M. \& Zavatta, A. Nonclassicality quasiprobability of single-photon-added thermal states. Phys. Rev. A 83, 032116 (2011).

40. Novoderezhkin, V. I., Yakovlev, A. G., van Grondelle, R. \& Shuvalov, V. A. Coherent nuclear and electronic dynamics in primary charge separation in photosynthetic reaction centers: a redfield theory approach. J. Phys. Chem. B 108, 7445-7457 (2004).

41. Hay, S. \& Scrutton, N. S. Good vibrations in enzyme-catalysed reactions. Nat. Chem. 4, 161-168 (2012)

42. Dasgupta, J., Frontiera, R. R., Taylor, K. C., Lagarias, J. C. \& Mathies, R. A. Ultrafast excited-state isomerization in phytochrome revealed by femtosecond stimulated raman spectroscopy. Proc. Natl Acad. Sci. USA 106, 1784-1789 (2009).

43. Turin, L. A spectroscopic mechanism for primary olfactory reception. Chem. Senses 21, 773-791 (1996).

44. Brookes, J. C., Hartoutsiou, F., Horsfield, A. P. \& Stoneham, A. M. Could humans recognize odor by phonon assisted tunneling? Phys. Rev. Lett. 98, 038101 (2007).

45. Franco, M. I., Turin, L., Mershin, A. \& Skoulakis, E. M. C. Molecular vibration sensing component in drosophila melanogaster olfaction. Proc. Natl Acad. Sci. USA 108, 3797-3802 (2011).

46. Davidovich, L. Sub-poissonian processes in quantum optics. Rev. Mod. Phys. 68, 127-173 (1996).

47. Scully, M. \& Zubairy, S. Quantum Optics (Cambridge University Press, 1997)

48. Klyshko, D. Observable signs of nonclassical light. Phys. Lett. A 213, 7-15 (1996).

49. Wong, C. Y. et al. Electronic coherence lineshapes reveal hidden excitonic correlations in photosynthetic light harvesting. Nat. Chem. 5, 396-404 (2012)

50. Hayes, D., Griffin, G. B. \& Engel, G. S. Engineering coherence among excited states in synthetic heterodimer systems. Science 340, 1431-1434 (2013).

51. Samsonoff, W. \& MacColl, R. Biliproteins and phycobilisomes from cyanobacteria and red algae at the extremes of habitat. Arch. Microbiol. 176, 400-405 (2001).

52. Scholes, G. D., Mirkovic, T., Turner, D. B., Fassioli, F. \& Buchleitner, A. Solar light harvesting by energy transfer: from ecology to coherence. Energy Environ. Sci. 5, 9374-9393 (2012).

53. Singh, D. K., Srivastava, S. K., Ojha, A. K. \& Asthana, B. ph-dependent raman study of pyrrole and its vibrational analysis using DFT calculations. Spectrochim. Acta Part A 71, 823-829 (2008).

54. Zhou, C., Diers, J. R. \& Bocian, D. F. Qy-excitation resonance raman spectra of chlorophyll a and related complexes. Normal mode characteristics of the lowfrequency vibrations. J. Phys. Chem. B 101, 9635-9644 (1997).

55. Vöhringer, P. et al. Solvent and frequency dependence of vibrational dephasing on femtosecond time-scales. J. Raman Spectrosc. 26, 535-551 (1995).

56. Kukura, P., McCamant, D. W. \& Mathies, R. A. Femtosecond stimulated raman spectroscopy. Annu. Rev. Phys. Chem. 58, 461-488 (2007).

57. Kühn, O., Renger, T. \& May, V. Theory of exciton-vibrational dynamics in molecular dimers. Chem. Phys. 204, 99-114 (1996).

58. Palma, G. M., Suominen, K.-A. \& Ekert, A. K. Quantum computers and dissipation. Philos. Trans. R. Soc. A 452, 567-584 (1996).

59. Merlo, M., Haupt, F., Cavaliere, F. \& Sassetti, M. Sub-poissonian phononic population in a nanoelectromechanical system. New J. Phys. 10, 023008 (2008).

60. Cavaliere, F., Piovano, G., Paladino, E. \& Sassetti, M. Phonon distributions of a single-bath mode coupled to a quantum dot. New J. Phys. 10, 115004 (2008).

61. Zavatta, A., Parigi, V. \& Bellini, M. Experimental nonclassicality of singlephoton-added thermal light states. Phys. Rev. A 75, 052106 (2007).

62. Cai, J., Popescu, S. \& Briegel, H. J. Dynamic entanglement in oscillating molecules and potential biological implications. Phys. Rev. E 82, 021921 (2010)

63. Silbey, R. \& Harris, R. A. Variational calculation of the dynamics of a two level system interacting with a bath. J. Chem. Phys. 80, 2615-2617 (1984).

64. Fassioli, F., Olaya-Castro, A. \& Scholes, G. D. Coherent energy transfer under incoherent light conditions. J. Phys. Chem. Lett. 3, 3136-3142 (2012).

65. Scholes, G. D., Fleming, G. R., Olaya-Castro, A. \& van Grondelle, R. Lessons from nature about solar light harvesting. Nat. Chem. 3, 763-774 (2012).

66. Schlau-Cohen, G. S. et al. Pathways of energy flow in LHCII from twodimensional electronic spectroscopy. J. Phys. Chem. B 113, 15352-15363 (2009). 
67. Dorfman, K. E., Voronine, D. V., Mukamel, S. \& Scully, M. O. Photosynthetic reaction center as a quantum heat engine. Proc. Natl Acad. Sci. USA 110, 2746-2751 (2013).

68. Tanimura, Y. \& Kubo, R. Time evolution of a quantum system in contact with a nearly Gaussian-markoffian noise bath. J. Phys. Soc. Jpn 58, 1017-114 (1989).

69. Ishizaki, A. \& Fleming, G. R. Unified treatment of quantum coherent and incoherent hopping dynamics in electronic energy transfer: reduced hierarchy equation approach. J. Chem. Phys. 130, 234111 (2009).

70. Shi, Q., Chen, L., Nan, G., Xu, R.-X. \& Yan, Y. Efficient hierarchical liouville space propagator to quantum dissipative dynamics. J. Chem. Phys. 130, 084105 (2009).

\section{Acknowledgements}

We thank Greg Scholes and Rienk van Grondelle for discussions. We are also grateful to Carles Curuchet for a preliminary analysis on the specific molecular origins of vibrational modes in the PE545 complex and to Avinash Kolli for his support on numerical calculations in the initial stages of this project. Financial support from the Engineering and Physical Sciences Research Council (EPSRC UK) Grant EP/G005222/1 and from the EU FP7 Project PAPETS (GA 323901)is gratefully acknowledged.

\section{Author contributions}

E.J.O. performed the calculations. A.O.-C. conceived, designed and supervised the research. Both authors analysed and discussed the results and co-wrote the manuscript.

\section{Additional information}

Supplementary Information accompanies this paper at http://www.nature.com/nature communications

Competing financial interests: The authors declare no competing financial interests.

Reprints and permission information is available online at http://npg.nature.com/ reprintsandpermissions/

How to cite this article: O'Reilly, E. J. and Olaya-Castro, A. Non-classicality of the molecular vibrations assisting exciton energy transfer at room temperature. Nat. Commun. 5:3012 doi: 10.1038/ncomms4012 (2014).

\section{(c) (1)}

This article is licensed under a Creative Commons Attribution 3.0 Unported Licence. To view a copy of this licence visit http:// creativecommons.org/licenses/by/3.0/. 